\title{
Creation of Laryngeal Grafts from Primary Human Cells and
}

\section{Decellularized Laryngeal Scaffolds}

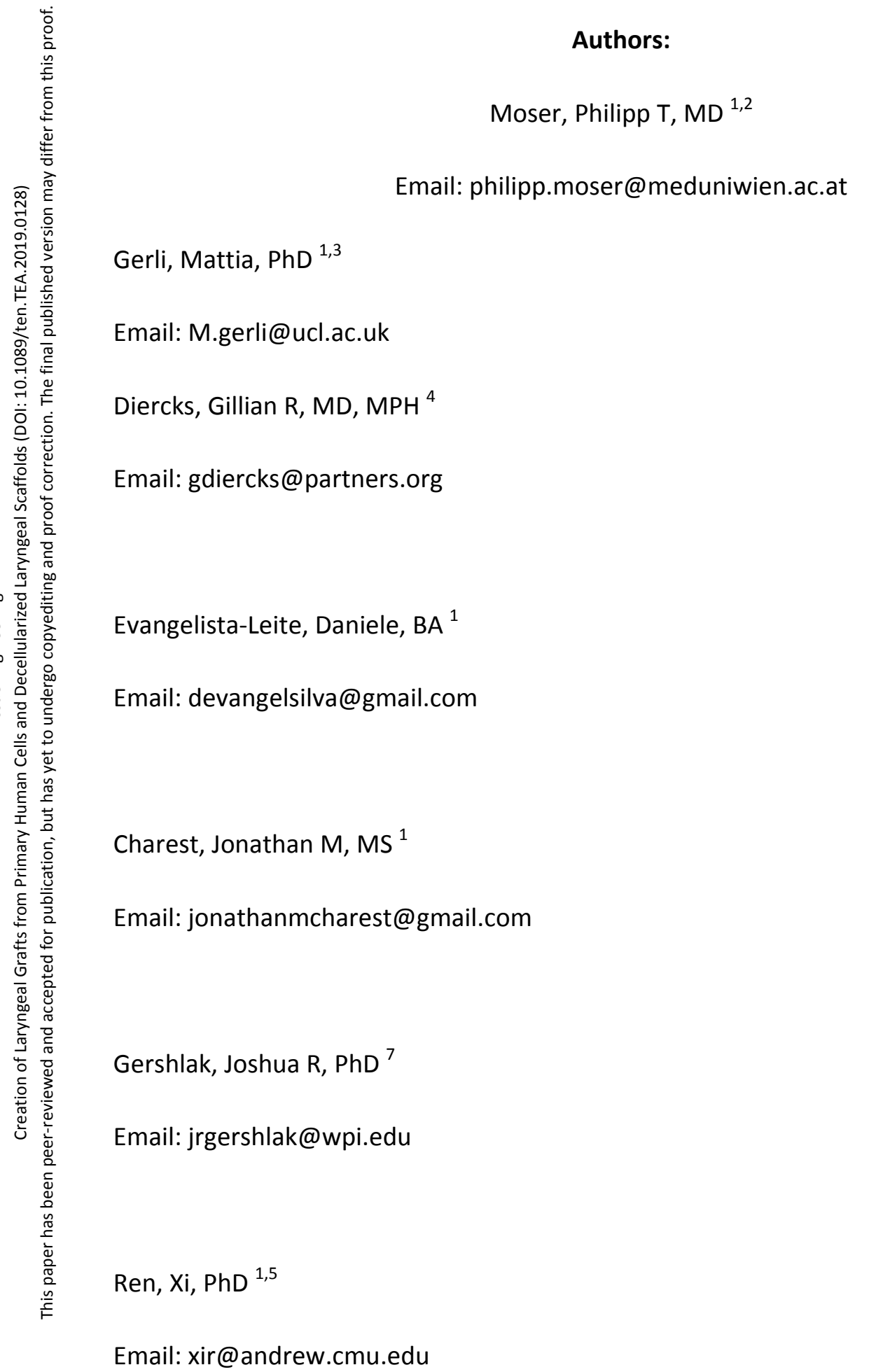


Gilpin, Sarah E, PhD ${ }^{1}$

Email: sgilpin@mgh.harvard.edu

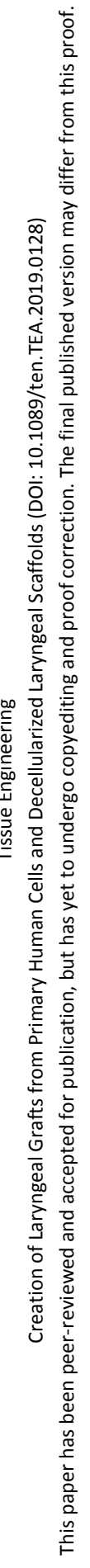

Jank, Bernhard J, MD ${ }^{1,6}$
Email: bernhard.jank@meduniwien.ac.at

Gaudette, Glenn R, PhD ${ }^{7}$

Email: gaudette@wpi.edu

Hartnick, Christopher J, MD, MS ${ }^{4}$

Email: christopher_hartnick@meei.harvard.edul

Ott, Harald C, MD ${ }^{1,8}$

Email: hott@mgh.harvard.edu

${ }^{1}$ Massachusetts General Hospital, Center for Regenerative Medicine 185 Cambridge St., Boston. 02114 MA, USA. P(617)643-0494 F(617) 724-2662

${ }^{2}$ Medical University of Vienna, Department of Biomedical Imaging and Image-guided Therapy

Spitalgasse 23, 1090 Vienna, AT. P004314040048180 F004314040048980

${ }^{3}$ University College London Medical School, Great Ormond Street Institute of Child Health London.

30 Guilford Street, WC1N 1EH London, UK. P02079052393 
${ }^{4}$ Massachusetts Eye and Ear Infirmary, Department of Otolaryngology.

243 Charles Street, Boston, 02114 MA, USA. P(617)573-3190 F (617) 573-6845

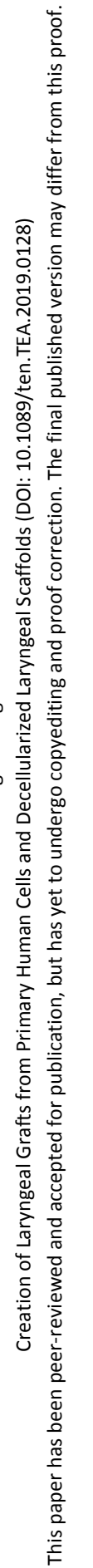

${ }^{5}$ Carnegie Mellon University, Department of Biomedical Engineering.

5000 Forbes Avenue, Pittsburgh, 15213 PA, USA. P(412) 268-7485 F(412) 268-3025

${ }^{6}$ Medical University of Vienna, Department of Otolaryngology.

Spitalgasse 23, 1090 Vienna, AT. P004314040033760 F004314040033320

${ }^{7}$ Worcester Polytechnic Institute, Biomedical Engineering.

60 Prescott Street, 01609 Worcester, MA, USA. P(978)821-7796

${ }^{8}$ Harvard Medical School, Department of thoracic Surgery.

55 Fruit Street, Boston, 02114 MA, USA. P(617)726-2311

Corresponding author and reprints: Harald C. Ott

Address: Ott lab for organ engineering, Richard B. Simches Research Center

MCPZN4700 185 Cambridge Street, Boston, MA 02116 P(617) 643-0494 F(617) 726-7667

Email:hott@partners.org 
ABSTRACT:

Current reconstruction methods of the laryngotracheal segment fail to replace the complex functions of the human larynx. Bioengineering approaches to reconstruction have been limited by the complex tissue compartmentation of the larynx. We attempted to overcome this limitation by bio-engineering laryngeal grafts from decellularized canine laryngeal scaffolds recellularized with human primary cells under one uniform culture medium condition.

First we developed laryngeal scaffolds which were generated by detergent perfusiondecellularization over nine days and preserved their glycosaminoglycan content and biomechanical properties of a native larynx. After subcutaneous implantations in rats for 14 days, the scaffolds did not elicit a CD3 lymphocyte response.

We then developed a uniform culture medium that strengthened the endothelial barrier over 5 days after an initial growth phase. Simultaneously, this culture medium supported airway epithelial cell and skeletal myoblast growth while maintaining their full differentiation and maturation potential. We then applied the uniform culture medium composition to whole laryngeal scaffolds seeded with endothelial cells from both carotid arteries and external jugular veins and generated re-endothelialized arterial and venous vascular beds. Under the same culture medium, we bio-engineered epithelial monolayers onto laryngeal mucosa and repopulated intrinsic laryngeal muscle. We were then able to demonstrate early muscle formation in an intra-muscular transplantation model in immuno-deficient mice. We supported formation of three humanized laryngeal tissue compartments under one uniform culture condition, possibly a key factor in developing, complex, multicellular, ready-to-transplant tissue grafts. 
IMPACT STATEMENT:

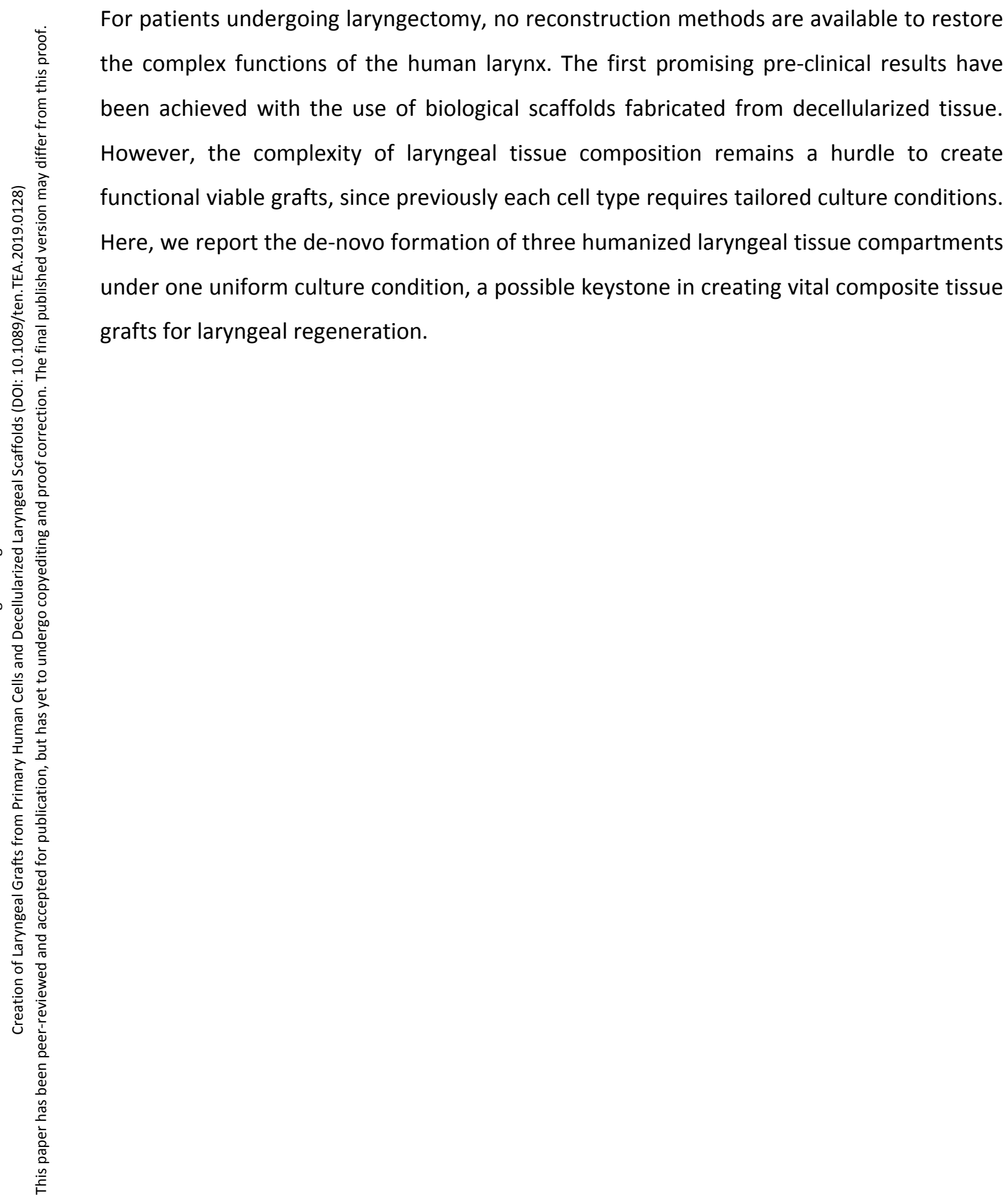


INTRODUCTION:

Hemi- or total-laryngectomy with permanent tracheostomy is often the only treatment option for laryngeal cancer or severe trauma. However, patients undergoing tracheostomy, experience a severe decline in quality of life and self-image $(1,2)$ The most devastating side effects are loss of voice and therefore of efficient communication, difficulties swallowing solid food and a loss of the sense of smell. In addition, long-term complications of tracheostomy include infections, trachea-esophageal fistula, erosion of the trachea and, narrowing or collapse of the tracheostomy (3). Currently, for patients undergoing laryngectomy there are no reconstruction methods available that restore the complex functions of the human larynx. Transplantation of native laryngeal allografts has been reported in detail, in two cases worldwide. $(4,5)$. However, the need for life-long immune suppression makes it a therapeutically irrelevant approach for most patients. The laryngeal architecture provides the foundation for swallowing, breathing and phonation, and therefore, laryngeal reconstruction based on the native cartilage framework may be a suitable approach. Generation of acellular laryngeal scaffolds with intact tissue architecture can be achieved by removal of the cellular components, yielding an acellular extracellular matrix (ECM) scaffold of low immunogenicity (6). To generate functional laryngeal tissue, scaffolds need to be revitalized, ideally with recipient derived cells. However, the complexity of laryngeal tissue composition remains a hurdle to bioengineering approaches, since previously each cell type requires tailored culture conditions. Here, we report the ex-vivo generation of laryngeal vasculature, epithelial mucosa and muscle-like tissue from human primary cells based on decellularized canine laryngeal scaffolds, under a single, uniform culture condition.

\section{MATERIALS AND METHODS:}

All procedures were performed in accordance with the National Institutes of Health guidelines for care and use of laboratory animals, and with approval of the Institutional Animal Care and Use Committee at the Massachusetts General Hospital. 
Isolation and perfusion-decellularization of whole larynxes:

Harvest of cadaveric larynxes was performed from beagles (24-36 months, $n=11$ ) post mortem. Briefly, a midline neck incision was performed, fascia and platysma were split, the common carotid arteries (CCAs) were located and cannulated with $16 \mathrm{G}$ cannulas (McMaster-Carr), followed by ligation of the internal and external carotid arteries.

Next, the proximal external jugular veins (EJs) were cannulated with $16 \mathrm{G}$ cannulas. The larynx was separated from surrounding tissue and explanted en bloc with the esophagus attached. We next tied off the esophagus cranially and caudally to the larynx and flushed the vasculature via the CCAs with heparinized saline for blood removal and to identify vascular leaks. Detected leaks were ligated with 3-0 silk suture. Larynxes were then mounted in a custom-built glass chamber and decellularization was initiated by perfusion from both CCAs in a single pass fashion. A constant pressure of $80 \mathrm{mmHg}$ was applied using a feedback control unit to regulate flow rate. Sequentially, larynxes were perfused with Phosphate-buffered saline (PBS) for 30 minutes, $1 \% \mathrm{v} / \mathrm{w}$ sodium dodecyl sulfate (SDS) for 5 days, deionized water for 2 hours, 1\% Triton-X 100 for 6 hours and finally washed with PBS for 4 days to allow the removal of the detergents from the matrix. All solutions were sterile and closely monitored for bacterial and fungal growth. All reagents were purchased from Sigma-Aldrich.

\section{Computer Tomography Angiography:}

Computer tomography scans were acquired through a dual-source CT scanner (SOMATOM Definition Flash, Siemens Healthcare). The decellularized whole larynx was mounted in a customized PTFE chamber and perfused with equal parts Visipaque contrast agent and $0.9 \%$ saline at a rate of $5 \mathrm{ml} / \mathrm{min}$ from both CCAs. Scanning was performed using a multiphase volume dynamic protocol and a three dimensional image was rendered from still images using MediCapture (MediCapture, Inc.;USA). 


\section{Biochemical Assays}

Biochemical analysis of native and decellularized canine larynxes were performed to determine the presence of glycosaminoglycan (GAG) and double-stranded DNA (dsDNA). Samples were divided in 3 groups: cartilage $(n=3)$, muscle $(n=3)$ and mucosa/submucosa $(n=3)$. Glycosaminoglycans (GAGs) analysis was performed using the Blyscan assay kit (Biocolor, UK). Dry samples were homogenized in $1 \mathrm{ml}$ of papain buffer containing $7 \mu \mathrm{l} / \mathrm{ml}$ of papain, digested at $65^{\circ} \mathrm{C}$ and incubated with the dye reagent. After washing, the samples were read in a 96 -well plate, at $656 \mathrm{~nm}$ and concentrations were calculated in reference to the relative standard curve. For dsDNA quantification, tissue samples were digested overnight at $55^{\circ} \mathrm{C}$ in $310 \mu \mathrm{L}$ of a solution containing $10 \mathrm{mM}$ Tris $(\mathrm{pH}=8.0), 5 \mathrm{mM}$ EDTA, $0.1 \mathrm{M} \mathrm{NaCl}, 1 \% \mathrm{SDS}$, and $650 \mu \mathrm{g} / \mathrm{mL}$ Proteinase-K (Life Technologies, Carlsbad, CA). dsDNA was then isolated using a phenol/chloroform extraction and alcohol precipitation, and quantified using the Quant-iT PicoGreen dsDNA kit (Life Technologies) following the manufacturer protocols. Fluorescence was read at $480 \mathrm{~nm} / 520 \mathrm{~nm}$ (excitation/emission) and concentrations were calculated in relation to the standard curve. All biochemical data are presented as the total mass of the component extracted normalized to tissue sample wet weight and normalized to dry weight (lyophilized tissue).

\section{Biomechanical testing}

For biomechanical testing, we compared the first tracheal ring of decellularized larynxes to native tracheal rings. Rings were cut into $1 \mathrm{~cm}$ long strips for uniaxial tensile testing, as previously described. Trachea strips were loaded into the grips of the Instron 5544 Uniaxial Tensile Tester Machine (Instron Inc. Norwood, MA) and were pulled to failure at a strain rate of $1.00 \mathrm{~mm} / \mathrm{min}$. During the tensile testing, trachea displacement was visualized using a FastTec HiSpec4 high-speed camera (FastTec Imaging Inc., San Diego, CA), with videos taken at 120 frames per second. Videos acquired were used to generate strain maps of the trachea. Average strain over the area was calculated using the High-Density Mapping software algorithm (7). High-Density Mapping measures sub-pixel displacement based upon a random light intensity object. The average strain was calculated and then visualized into a heat map showing the localization of strain during the tensile testing. 


\section{Histological stains}

Tissue samples were fixed overnight in 4\% Paraformaldehyde (PFA, Sigma Aldrich), paraffin-embedded, and sectioned at 5-7 um thickness. Histological assessment was performed using standard protocols for the following stains: Hematoxylin/ Eosin (Sigma Aldrich), Mason's Trichrome (American Master Tech), Mason Pentachrome (American Master Tech), Safranin-O/ Fas green stain, and Toluidine blue stain. For immunofluorescent analysis, we performed antigen retrieval on deparaffinized sections using a citrate-based antigen unmasking solution (Vector Laboratories; H-3300). The sections were blocked with 1\%BSA (Sigma Aldrich) and incubated overnight at $4^{\circ} \mathrm{C}$ with the following primary antibodies: Rabbit (rbt) anti-LamAC (Abcam, ab108595), rat anti-KI67 (eBioscience; 14-5698), rbt anti-Collagen IV (Abcam; ab6586), ms anti-CD3 (Abcam; ab16669), ms anti-CD68 (AbD Serotec; MCA341GA), rbt anti-CD163 (Bioss, Inc; bs-2527R), rbt anti-CD80 (Bioss, Inc; bs-2211R), goat anti-E-Cadherin (R\&D Systems; AF648SP), rbt anti-CK5 (Abcam; ab52635), ms anti-P63 (Biocare Medical; CM163A), ms anti-acetyl K40 (Abcam; ab24610), rbt anti-CCSP (Abcam; ab40873), ms anti-MU5AC (Abcam; ab3649), ms anti-Fox J1(eBioscience; 14-9965-82), ms anti-CD31 (DAKO; M082301-2), rbt anti-VE-CAD, ms anti-MYH (DSHB; MF20), ms anti-eMYH (DSHB; F1.652). After primary antibody binding, the sections were washed and incubated for 40 minutes at room temperature with the appropriate donkey-raised secondary antibodies, conjugated with a 488, 546 or 647 fluorochromes (Life Technologies; AlexaFluor series) at a dilution of 1:400 in BSA. For apoptosis detection in combination with immunofluorescence staining, slides were first incubated with primary and secondary antibody, as described above, followed by apoptosis detection with Fluorometric TUNEL System (Promega).

\section{Immunologic response to decellularized xenografts}

Donor tissue preparation: Segments of proximal tracheal rings were isolated from native or perfusion-decellularized laryngo-tracheal constructs and divided in pieces of similar size. Recipients: Nine male SD rats (275-300 g) were randomly assigned to 3 groups: NAT, $n=3$; DEC, $n=3$ and Sham, $n=3$. Animals were anesthetized using an induction chamber and maintained with 1-3\%. A subcutaneous $1.5 \mathrm{~cm}^{2}$ pocket was created the midline oft the 
back of all animals. The donor tissue was placed in the pocket and the incision was closed with 4-0 silk. Animals of the Sham group did not receive a tissue implant. For blood count analysis, whole blood samples were collected from the tail vein with $1 \mathrm{ml}$ lithium heparin tubes (Greiner Bio-One $\mathrm{GmbH}$ ) on day 5 after implantation. On day 14 animals were sacrificed, the implants were carefully removed, leaving equal amount of fascia and skin attached, and fixed in PFA overnight. To assess cell infiltration, serial sections of the embedded tissue specimens were cut at $5 \mu \mathrm{m}$ thickness. The tissue sections were immunohistochemically stained for CD3, CD68, CD80 and CD163. Five representative fields per specimen were imaged at 20x magnification and used for analysis. A CellProfiler image analysis pipeline was used to quantify CD3+ and CD68+ cells, as well as macrophage subpopulation M1/M2 (8) In total, more than 60000 cells were analyzed across 3 groups containing 15 images each.

\section{Human primary cell isolation, characterization and expansion}

Human basal cells ( $\mathrm{hBCs}$ ) were isolated from donated human lungs, not suitable for transplantation and provided by the New England Organ Bank. Tracheal segments were freed from surrounding tissue and transferred into minimum essential medium (MEM) containing $140 \mathrm{mg}$ pronase (Sigma-Aldrich) and $10 \mathrm{mg}$ DNase (Sigma-Aldrich) per $100 \mathrm{~mL}$ and incubated at $4^{\circ} \mathrm{C}$ for 24 to 48 hours under constant agitation. The harvested cell suspension was transferred to polypropylene tubes, centrifuged at $300 \mathrm{~g}$ for 5 minutes, resuspended in MEM containing 20\% Fetal bovine Serum (FBS) and repeated. After the third centrifugation step the resulting cell suspension was resuspended in Small Airway Epithelial Growth Medium (SAGM, Lonza) supplemented with Rho-associated protein kinase inhibitor $(10 \mu \mathrm{M})$ and plated on non-coated tissue culture plastic to enrich the epithelial cell population. Cells were then transferred to previously $10 \mu \mathrm{g} / \mathrm{mL}$ collagen IV coated flasks and culture medium was changed to SAGM from the second day. Between passage 1 and 2 differential plating was used for further epithelial cell enrichment.

At passage 2 cells were characterized using immunofluorescence staining of P63 and Ecadherin and used for further experiments. 
Primary human myoblasts ( $\mathrm{hMBs}$ ) were isolated from anonymized skeletal muscle biopsies and fragmented into pieces below $0.5 \mathrm{~mm}$ and digested twice in $20 \mathrm{ml}$ of $0.005 \%$ trypsin $0.1 \%$ Collagenase D. The so obtained cell suspension was pre-plated on tissue culture dishes for 1 hour to enrich for the myoblast fraction over the highly adherent fibroblasts. The supernatant was then seeded for one week on Matrigel coated plates and characterized via FACS to validate cell identity. For FACS analysis, cells were trypsinized, blocked and resuspended in PBS 1\% FBS 0.05mM EDTA with the following antibodies: CD56 (Biolegend 5.1H11), CD31 (Biolegend WM59), CD146 (Biolegend P1H12). Cells were cultured in Medium referred to as myoblast growth Medium (MGM) as follows (9): Megacell 5\%FBS, 1\% P/S, 1\% NEAA, 0.1mM 2-mercaptoethanol, 5ng bFGF.

Human umbilical vein endothelial cells (HUVECs, Lonza) were maintained in $0.1 \%$ gelatincoated (Sigma-Aldrich) flasks with complete EGM-2 (Lonza).

\section{Cell culture tests}

HUVECs (100k/6 well, gelatin coated), hBCs (100k/6 well, collagen IV coated), and hMBs (100k/6 well) were plated using conventional EGM-2, SAGM and MGM, respectively. After complete cell attachment, cells were rinsed with PBS three times and the culture medium was switched to the following 3 conditions per cell type: EGM-2, SAGM, MGM ( $n=3$ per condition and cell type). Media changes were performed every other day. On day 5, HUVECs were methanol fixed and stained for PECAM-1 and VE-cadherin; hBCs were methanol fixed and stained for P63, E-cadherin; bright field images of myoblasts were taken.

\section{Evaluation of one uniform culture condition: Multi Cell Growth Medium}

For MCGM testing on HUVECs the following steps were taken: HUVECs were plated onto gelatin coated 12-wells in EGM-2 containing 10\% FBS (EGM2-10\%). On day 3, confluent HUVEC monolayers were rinsed with PBS three times and the culture medium was replaced by multi cell growth medium (MCGM): SAGM + Medium 199 (Gibco) (1:1) supplemented with $5 \%$ fetal bovine serum (FBS), 0.5\% Insulin-Transferrin-Selenium (Gibco), ascorbic acid ( $25 \mu \mathrm{g} / \mathrm{mL}$; STEMCELL Technologies), recombinant human vascular endothelial growth factor (VEGF) $(20 \mathrm{ng} / \mathrm{mL})$, (bFGF) $(20 \mathrm{ng} / \mathrm{mL})$, and $1 \%$ 
antibiotic/antimycotic solution. Cells were maintained in MCGM for 5 days with medium changes performed every other day. HUVECs cultured in EGM2-10\% for 8 days served as controls. On day 8 cells were methanol fixed and stained for PECAM-1, VE-Cadherin.

hBCs were plated onto collagen IV coated 12-wells in MCGM and maintained for 5 days with alternate day media changes. On day 5 cells were methanol fixed, and stained for Ecadherin, CK5 and P63. hBCs cultured in SAGM for 8 days served as control. For hBC differentiation, cells were plated onto $0.4 \mu \mathrm{m}$ transwell inserts coated with collagen IV and maintained in submerged culture with MCGM for 5 days. Media was replaced with PneumaCult ${ }^{\mathrm{TM}}$-ALI medium (Stemcell Technologies) in the basal chamber only, and maintained for 28 days at Air-Liquid interface (ALI) (10). Media changes were performed every other day.

At the end of ALI culture inserts were PFA fixed, paraffin embedded and stained for the following markers: E-cadherin, CK5, P63, ACT TUB, FOX J1, MUC 5AC and CCP.

hMBs were plated onto 12-wells in MCGM and maintained for 5 days with media changes every other day. On day 5 hMBs were rinsed in PBS three times, the culture medium was replaced by myoblast differentiation medium (DMEM, 2\% Horse serum, 1\% P/A, 1\%NEAA) and cells were differentiated for 14 days with medium changes every other day. hMBs were cultured in conventional MGM for 8 days, followed by conventional myoblast differentiation medium as controls. On day 14 of differentiation cells were methanol fixed and stained for MYC.

\section{Cell proliferation assay}

Quantitative assessment of HUVEC proliferation under EGM2-10\% and MCGM was performed as follows: HUVECs were plated onto gelatin coated 6-wells at a density of $10 \mathrm{k} /$ well and maintained for 5 days in EGM-2, EGM2-10\% and MCGM with medium changes performed on alternate days. At day 5 cells from 3 wells per condition were counted using an automated system. Cell numbers are normalized to counted cells in EGM-2. 


\section{Cell differentiation assay}

For quantitative assessment of hMB differentiation, the percentage of Myosin heavy chain (MYH) positive cells in groups treated with MCGM or conventional MGM ( $n=6$ per condition) for 5 days, followed by 14 days of differentiation was calculated using Image J Software (NIH,USA)

\section{Dextran transwell permeability assay}

We measured dextran permeability across the HUVEC monolayer using $3.0 \mu \mathrm{m}$ pore Transwell inserts (12 well format). HUVECs were plated on gelatin coated inserts at a density of 75,000 cells/well and cultured, submerged in EGM2-10\% for 3 days, followed by 5 days of MCGM. HUVECs cultured in EGM2-10\% for 8 days served as controls ( $n=3$ per condition). Completing the culture, $50 \mu \mathrm{l}$ of FITC-conjugated $500-\mathrm{kDa}$ dextran $(5 \mathrm{mg} / \mathrm{ml}$, Sigma-Aldrich) was added to the upper chamber and the media fluorescence of the lower chamber was measured after 30 min of incubation at $37^{\circ} \mathrm{C}, 5 \% \mathrm{CO} 2$ using SpectraMax Microplate Reader (Molecular Devices) set at $485 \mathrm{~nm}$ (excitation)/538 nm (emission). Fluorescence readings were normalized to dextran permeability in transwell inserts without HUVECs.

\section{Whole larynx endothelial cell seeding}

Decellularized laryngeal scaffolds $(n=3)$ were primed by perfusion from both the right and left CCA and right and left EJ at $2 \mathrm{ml} / \mathrm{min}$ with $100 \mathrm{ml}$ of EGM2-10\% for 24 hours.

80 million HUVECs were suspended in $400 \mathrm{ml}$ EGM2-10\%, and seeded simultaneously through the four vascular accesses (100 $\mathrm{ml}$ each) at $100 \mathrm{mmHg}$ (CCAs) and 60mm Hg (EJs). After 2 hours of static culture to allow cell attachment, the culture medium was filtered to remove non-adherent cells and the laryngeal scaffold was perfused from all 4 vascular accesses at $1 \mathrm{ml} / \mathrm{min}$ medium for 2 hours, followed by $2 \mathrm{ml} / \mathrm{min}$ perfusion rate for 3 days. Subsequently, on day 3, the vascular ports of left and right EJ were removed (open to chamber) and the scaffold was perfused from arterial side only, using $200 \mathrm{ml}$ of MCGM. Medium changes were performed on alternate days. On day 8 of overall culture, larynxes were removed from the seeding chamber and PFA fixed by perfusion. 


\section{In vitro reconstruction of epithelialized mucosa}

For re-epithelialization of acellular laryngeal muscle constructs, we first isolated the luminal mucosa from the decellularized scaffolds using a $6 \mathrm{~mm}$ punch biopsy. Mucosa was primed in MCGM for 24 hours and then seeded with hBCs at a density of 700,000 cells/ $\mathrm{cm}^{2}$. Two hours after seeding cell suspension was replaced with new MCGM and constructs were cultured for 5 days with culture medium changes performed daily. On day 5 constructs were PFA fixed for histology $(n=6)$.

\section{In vitro reconstruction of laryngeal muscle}

Laryngeal intrinsic muscle ECM was isolated from perfusion-decellularized larynxes and primed in MCGM for 24 hours. For seeding, 2 million hMBs were re-suspended in $100 \mu \mathrm{l}$ MCGM and injected along the fiber direction into the scaffold, using a 29G insulin syringe (Exelint). Repopulated constructs were cultured in MCGM for 24 hours under static condition. From day one static culture was replaced by dynamic condition using an orbital shaker (Troemner, NJ) and medium was replaced daily. On day 5 of culture constructs were either PFA fixed for histology or used for in vivo experiments.

\section{D Viability and Proliferation assay}

To assess the viability of in vitro generated muscle constructs and increase in cellularity, a modified Resazurin (Presto blue, Invitrogen) reduction assay was performed on day 1 and day 5. (11) Muscle constructs were transferred to 12-wells and incubated in $1 \mathrm{ml}$ MCGM containing $50 \mu \mathrm{l}(1: 20)$ resazurin for 60 minutes at 37 degrees under constant agitation. The fluorescence intensity of the metabolized medium was read using a SpectraMax Microplate Reader (Molecular Devices, Sunnycale, CA) at $544 \mathrm{~nm}$ (ex), $590 \mathrm{~nm}$ (em). Unseeded scaffolds served as controls.

\section{In vivo maturation of regenerated laryngeal muscle}

In vitro regenerated muscle constructs $(n=6)$ were transplanted into 6-weeks old, female Nude mice. Under general anesthesia, a $1.5 \mathrm{~cm}$ skin incision was performed on the anterolateral side of the lower hind limb and the fascia of the tibialis anterior muscle was carefully lifted from the underlying muscle. The regenerated muscle construct was then 
placed between fascia and muscle, thereby matching the fiber direction of the native muscle.(12) The skin incision was closed with 6-0 silk (Ethicon), the animals were weaned from anesthesia and single housed. Animals receiving regenerated muscle implants showed no signs of limping or reduced physical activity on day 1 after implantation. Bioengineered muscle constructs were explanted from sacrificed animals on day 14 after implantation, fixed with PFA and embedded in paraffin for histology.

\section{Statistical tests}

Student t-tests and one-way analysis of variance followed by Bonferroni multiple comparison tests were performed using PRISM 6.

\section{RESULTS:}

\section{Creation of native laryngeal scaffolds.}

We isolated whole canine larynxes post mortem and proceeded with the cannulation of the vasculature. The larynxes were then mounted in an ad-hoc designed perfusion chamber. We performed pressure controlled perfusion-decellularization through both common carotid arteries using an adapted detergent based protocol. (13) Over 5 days of $1 \%$ sodium dodecyl sulfate (SDS) perfusion, followed by 6 hours deionized water and 6 hours $1 \%$ Triton $\mathrm{X}-100$, the composite tissue gradually lost opacity as decellularization occurred. Perfusion-decellularization followed by clearing of detergents was completed on day 9 (Fig. 1A). Macroscopically, the vocal fold turned transparent and on gross examination appeared to be edematous. A thin, yellow mucosal layer covered the luminal surface of the larynx. Epiglottis, cricoid, and thyroid cartilage retained their shape (Fig. 1B). We confirmed vascular continuity after decellularization with a Computer Tomography Angiography (CTA) (Fig. 1C).

DAPI staining confirmed successful removal of the nuclei from the laryngeal mucosa, exocrine glands, perichondrium and the outer layer of the cartilage (Fig. 1D, left panel). These findings were supported by a substantial decrease in double stranded DNA (dsDNA) found in mucosa / submucosa, muscle and hyaline cartilage of decellularized (DEC) 
compared to native (NAT) specimens (dsDNA ng/mg dWT; NAT vs. DEC: Mucosa: $654.6 \pm 227.7$ vs. $115.8 \pm 38.8, p=0.0156$; Muscle: $966.3 \pm 316$ vs. $282.7 \pm 92.32, p=0.0228$; Cartilage: $141.1 \pm 20.01$ vs. $53.93 \pm 21.32, p=0.0067$ ) (Fig. $1 D$, right panel). Hematoxylin \& Eosin (HE) stain of an axial laryngeal section showed preservation of laryngeal microarchitecture (Fig. 1E). The ultrastructure of the decellularized submucosa, vasculature, muscle and cartilage, was assessed by Russel-Movat pentachrome stain. The observed tissue compartments remained intact and showed loss of cellular components (Fig. 1F).

\section{Biochemical and biomechanical characterization of the laryngeal scaffold.}

Glycosaminoglycan (GAG) occupies the interfibrillar space of the cartilage ECM provides cartilage with its osmotic properties, which are critical to its ability to resist compressive loads. (14). GAGs were preserved and equally distributed throughout the cartilage, as assessed qualitatively by Safranin-O / Fas green and Toluidine Blue stain (Fig. 1G). Analysis of the GAG content did not reveal a significant difference between native and decellularized cartilage (GAG content in $\mu \mathrm{g} / \mathrm{mg}$ : NAT $376.4 \pm 89.36$ vs. DEC 291.5 \pm 85.11 ; $p=0.122$, Fig. 1G). To determine the impact of decellularization on the biomechanical properties of cartilage, we performed strain testing. Decellularized cartilage underwent non-significant changes in strain over the area of tested tissue (Strain over strain map: NAT $0.228 \pm 0.0519$ vs. DEC 0.2742 0.0194; $p=0.2488$, Fig. $1 \mathrm{H}$ )

\section{Immunological response to laryngeal scaffolds.}

To evaluate the immunogenicity of the decellularized scaffolds, we subcutaneously implanted native and decellularized canine tracheal tissue into immune-competent rats for 14 days (Fig. 2A). Sham operated animals did not receive implants and served as controls. On day 5, white blood cell count showed a significant increase of monocytes in animals receiving native tissue. (Monocytes in $10^{3} / \mu \mathrm{l}$ : 0.93 \pm 0.25 NAT vs. $0.46 \pm 0.11 \mathrm{DEC}$ vs. Sham $0.5 \pm 0.26$; NAT vs. DEC, $p<0.005$, NAT vs. Sham, $p<0.005$, DEC vs. Sham, $p=n s ;$ ). Lymphocyte, granulocyte and total white blood count did not significantly differ between groups (Fig. 2B). On day 14, we removed composite tissue implants and evaluated for cellular infiltration by histology. Both DEC and NAT implants had induced angiogenesis, 
likely due to a foreign body inflammatory response (Fig. 2A). Consistent with macroscopic findings, histology revealed blood perfused vasculature at the border zone between recipient tissue and implant (Fig. $2 \mathrm{C}$ left panel). In addition we saw neo-vascularization in the mucosa of DEC implants. NAT implants showed diffuse infiltration of $\mathrm{CD}^{+}$lymphocytes in the mucosa and submucosa, whereas we observed limited lymphocyte migration to the cartilage (Fig. 2D left panel). In contrast, we detected minimal infiltration of $\mathrm{CD}^{+}$cells in the DEC mucosa and submucosa, and absence of $\mathrm{CD}^{+}$cells in the cartilage. (Fig. $2 \mathrm{C}$ left panel). We analyzed over 60,000 cells, using a CellProfiler image analysis pipeline, for expression of CD3 (Fig. 2C right panel) and found $69.7 \pm 14.3 \%$ to be positive in NAT, $6.1 \pm 2.2 \%$ in DEC, and $3.5 \pm 2.4 \%$ in Sham. (NAT vs. DEC: $p<0.0001$; NAT vs. Sham: $p<0.0001$; DEC vs. Sham, $p=0.533)$. The total number of CD3+ cells per field were: $1276 \pm 418$ NAT, $30 \pm 4$ DEC, and 10 \pm 6 Sham (NAT vs. DEC: $p<0.0001$; NAT vs. Sham: $p<0.0001 ;$ DEC vs. Sham, $p=0.9030)$. We next assessed macrophage (CD68+) infiltration (Fig. 2D). Within implants $43.9 \pm 6 \%$ of cells were CD68+ in NAT, $11.7 \pm 1.5 \%$ in DEC and $18.3 \pm 1.6 \%$ in Sham (NAT vs. DEC: $p<0.0001$; NAT vs. Sham: $p<0.0001 ;$ DEC vs. Sham, $p=0.1950)$. The total number of $\mathrm{CD}^{+} 8^{+}$cells per field were $768 \pm 197$ in NAT, $44 \pm 19$ in DEC, and $60 \pm 25$ in Sham (NAT vs. DEC: $p<0.0001$; NAT vs. Sham: $p<0.0001$; DEC vs. Sham, $p=0.911$ ). To assess the macrophage response to implanted decellularized tissue in more detail, we looked for macrophage type 1 (M1: $\mathrm{CD}^{+} \mathrm{CD} 80+$ ) and type 2 (M2: CD68+ CD163+) polarization (Fig. 2F). While M1 predominance is associated with dense tissue deposition and scarring, M2 results in a more constructive type remodeling (8). Immune histological stains revealed a high density of CD68+ cells in NAT specimen with a dominance of M1 macrophages over M2 (Fig. 2E, left panel). M1 macrophages contributed to $82.6 \pm 15.1 \%$ of CD68+ cells in NAT, compared to $37 \pm 15.4 \%$ in DEC ( $p=0.0196)$. M2 macrophages contributed to $48.7 \pm 20 \%$ of CD68+ cells in NAT, compared to $41.2 \pm 9.1 \%$ in $\mathrm{DEC}(\mathrm{p}=0.5906)$ (Fig. $2 \mathrm{E}$, right panel). Overall, native tissue showed a clear M1 predominance whereas no distinct macrophage phenotype was found in decellularized tissue implants. 


\section{Development of a uniform culture condition for bioengineering of laryngeal grafts.}

To repopulate scaffolds, we chose three primary human cell types. Human umbilical endothelial cells (HUVECs) were used for re-endothelialization of whole scaffolds based on a previously described two-step seeding protocol (15). We obtained human epithelial cells from fresh cadaveric tracheas for re-epithelialization of the laryngeal mucosal ECM (Fig. 3A). Epithelial-oriented culture yielded a uniform human epithelial basal cell ( $h B C)$ population, characterized by immunofluorescence stain for E-Cadherin and P63 (Fig. 3B). Additionally, we isolated primary human myoblasts from discarded muscle biopsies through a mechanical/enzymatic method (Fig.3A). We subsequently cultured the cells at low density in myoblasts media (Fig. 3C, left panel) and characterized the cell population by three-marker flow cytometry. Isolated cells were positive for CD56 and negative for CD31 and CD45, consistently with the expected cell phenotype (Fig.3B, right panel).

To allow for repopulation of laryngeal scaffolds with primary human cells, we explored a cell culture medium composition that maintains and matures pre-formed endothelial structures, while supporting engraftment and growth of hBCs and hMBs for laryngeal tissue formation.

In a pilot study, we tested conventional endothelial, epithelial and myoblast cell culture media on all three cell types (Supp. Fig. 1). As expected neither of the media conditions tested could maintain cell phenotype and/or cell survival of all three different cell types. We chose a composition based on conventional airway epithelial medium, since hBCs were most difficult to maintain in absence of its medium and modified it to support endothelial and myoblast culture. This medium is referred to as Multi-Cellular Growth Medium, (MCGM). For the two-step endothelial cell seeding process, we first cultured HUVECs in conventional endothelial growth medium containing 10\% fresh frozen plasma (EGM2-10\%) until confluency was reached on day 3 (Growth phase), followed by 5 days of MCGM (Maturation phase) (Fig. 3D). After contact inhibition, monolayers could be maintained without cell detachment, loss of cell phenotype and with strong cell-cell adhesion, as revealed by immunofluorescence stain for PECAM-1 and VE-Cadherin (Fig. 3D). HUVECs cultured in EGM2-10\% for 8 days served as a control (Supp. Fig. 2A). In addition, MCGM lead to a significant reduction in cell proliferation, compared to EGM2-10\%. (Cell 
proliferation rate normalized to EGM-2: EGM2-10\% 1.29 \pm 0.04 vs. MCGM $0.43 \pm 0.073$; $p=0.0004 ;$ Fig 3F, left panel) and confluent monolayers formed a stronger intercellular barrier in MCGM, compared to EGM2-10\% (500 kDa dextran leakage (\%): MCGM $0.0065 \pm 0.0062$ vs. EGM2-10\% 0.46 $\pm 0.031 ; p<0.001$ ) (Fig. 3D, lower panel)(16).

We then evaluated the effect of MCGM on hBCs over 5 days of culture (Fig. 3G). hBCs formed a continuous E-cadherin monolayer and cells homogeneously expressed the basal cell marker P63 and Cytokeratin 5 (Fig. 3H, upper panel). hBCs cultured in conventional epithelial medium for 5 days served as control (Supp. Fig. 2B). To confirm maintenance of differentiation potential, basal cells were cultured in MCGM for 5 days and then differentiated under air/liquid interface culture for 28 days. Cells formed a pseudostratified epithelium composed of a basal cell layer (Fig. $3 \mathrm{H}$, lower panel). In addition, ciliated cells (Acetyl. alpha tubulin ${ }^{+}$, Fox $^{+}{ }^{+}$), goblet cells (Mucine $5 \mathrm{AC}^{+}$), and club cells $\left(\mathrm{CCP}^{+}\right)$were present in the newly formed epithelium (Fig. 3I).

Myoblasts cultured in MCGM vs. conventional myoblast growth medium, followed by 14 days of differentiation showed no phenotypical difference (Fig.3J and Supp. Fig. 2C). We evaluated myoblast differentiation as the percentage of nuclei contributing to $\mathrm{MYH}^{+}$fibers, resulting in a significantly higher percentage in MCGM than in conventional myoblast growth medium $\left(\mathrm{MYH}^{+}\right.$fibers in \%: MCGM 18.36 \pm 6.4 vs. conventional medium $10.9 \pm 4.49$, $p=0.041$ ). Once an optimal uniform culture condition for all three primary cell types was identified we applied it to bioengineered laryngeal tissue.

\section{Bioengineering of laryngeal grafts (under one uniform culture condition).}

The first step in generating vital laryngeal tissue was the development of an endothelialized vascular network within the whole laryngeal scaffold. With this aim, the larynxes were mounted in a custom designed bio-chamber (Fig. 4A) and seeded with a total of 80 million HUVECs. We have shown that HUVEC seeding from both arterial and venous side significantly benefits endothelial cell coverage (15). Thus, we chose to deliver cells, simultaneously, through four vascular accesses: the right and left common carotid arteries and the right and left external jugular veins (Fig. 4A, right panel). We perfusioncultured whole larynxes for 3 days in EGM2-10\% to promote HUVEC proliferation. We then applied MCGM culture to endothelialized laryngeal scaffolds to further mature the 
endothelium and complete the endothelialization process. Immunofluorescence imaging revealed a high density of PECAM- $1^{+}$vascular structures within the native ECM (Collagen IV) scaffold (Fig. 4B). We observed endothelial lining in both arterial and venous vascular beds, suggesting successful delivery of cells from arterial and venous vascular accesses. Notably, endothelial delivery reached vascular segments of different calibers. (Fig. 4C). This indicates endothelial lining of the hierarchical vascular network.

For re-epithelialization, we seeded about 700,000 cells $/ \mathrm{cm}^{2}$ onto the decellularized mucosa of laryngeal grafts and cultured them for tissue formation in MCGM for 5 days. Immuno-histologic characterization showed that hBCs were engrafted to the luminal surface of Collagen IV positive basal membrane of the mucosal ECM, preferentially forming a continuous monolayer (Fig. 4D). The basal cell layer remained positive for E-cadherin and CK5 under MCGM culture (Fig. 4E). Based on the results from the previous cell culture experiments, the newly formed laryngeal mucosa can likely be differentiated into mature pseudostratified epithelium.

To repopulate the laryngeal intrinsic muscular ECM, we seeded a total of 2 million myoblasts per $100 \mu$ injection volume into laryngeal intrinsic muscular ECM and cultured the tissue for 5 days in MCGM. To trace cell viability, in cell culture medium, we measured fluorescence of metabolized resazurin, a surrogate for cell viability and growth (11). Resazurin metabolism revealed a significant increase in fluorescence over 5 days, indicating cell proliferation (d1 $1190 \pm 246.7$ vs. d5 2235 \pm 580.3 ; p=0.0165) (Fig. 4F). Immuno-histologically, human Lamin A/C (LamAC+) identified human cells densely repopulating the scaffolds on day 5 of culture (Fig. 4G).

In vivo maturation of bioengineered laryngeal grafts.

To investigate whether active differentiation to muscle-like tissue can be achieved in vivo, we implanted the in vitro engineered laryngeal intrinsic muscle grafts into nude mice. We chose the sub-fascial space of the anterior tibialis muscle for implantation because it provides mechanical stimulation and is easily accessible $(2,12)$ (Fig. 5A). On day 14 postimplantation, trichrome stain of cross-sections from explanted tissue revealed a defined area of muscle-like tissue within the implant (Fig. 5B). Immunofluorescence images showed the distribution of human identified cells $\left(\operatorname{LamAC}^{+}\right)$within the implanted scaffold 
(Fig. 5C, left panel). Absence of TUNEL indicated viability of LamAC cells in the bioengineered muscle constructs. As to be expected there was little proliferation of LamAC+ cells during the stage of maturation. (Fig. 5C, right panel). In contrast to day 5 of ex vivo culture, early developmental fiber formation could be observed (Fig. 5D) and a broad myosin expression of LamAC+ cells, as well as, formation of multinucleated fibers was found. (Fig. 5E, lefty panel). The presence of embryonic myosin confirmed an early developmental phenotype (Fig. 5E, right panel). We did not see contribution of recipient myoblast ( LamAC $^{-} /$Myosin $^{+}$) to muscle formation within implants. In addition, LamAC $^{+}$cells were not found beyond the border of the implant (Supp. Fig. 3). Finally, fibers of organized myosin striation were present in the newly formed muscle-like laryngeal tissue. (Fig.5F)

\section{DISCUSSION:}

The human larynx is a highly orchestrated organ, with several tissue compartments contributing to the function of swallowing, breathing and phonation. In order to achieve laryngotypic organ function various tissue compartments are necessary. Here, we describe the decellularization of whole larynxes by vascular perfusion, on a clinically relevant scale. We showed ex vivo formation of laryngeal tissue compartments from three primary human cell types under one uniform culture condition and assessed in vivo compatibility and tissue maturation. Immersion decellularization of human larynxes has been achieved by 25 consecutive cycles of a detergent-enzymatic method, which reportedly took several weeks (17). More recently, acellular laryngeal scaffolds were generated from pigs within 8 days using negative pressure in combination with freeze-thawing and hyper-hypotonic solutions, followed by detergent-enzymatic immersion (18). While this method reduced the processing time significantly, a loss of mechanical durability of the laryngeal cartilage was reported and attributed to a substantial loss in GAG content during the decellularization process. With our selective perfusion protocol, we minimized exposure of detergents to 5 days, which efficiently eliminates immunogenic properties and preserves the critical GAG content and biomechanical properties of the avascular cartilage. The immunologic profile of implanted decellularized scaffolds revealed a nonspecific foreign body reaction and negligible antigen mediated immune response, comparable to sham procedures. In contrast, native tissue showed significant lymphocyte and M1 dominant 
infiltration. Notably, despite remaining chondrocytes within the cartilage, no $\mathrm{CD}^{+}$cells infiltrated the cartilage, nor were recruited to the perichondrium. These findings agree with previous reports of possible immune evasion of laryngeal cartilage due to low MHC II expression (19). Development of true laryngeal tissue is challenging due to the vast number of cell types that resident within the organ, each with special requirements for culture conditions. In this work, we propose a uniform culture condition to support development of laryngeal grafts from three human primary cell types ex vivo. A cell culture medium that supports endothelial maturation as well as cell engraftment and proliferation of epithelial cells and myoblasts is a useful tool for ex vivo laryngeal tissue assembly. By using four vascular accesses for cell delivery we achieved hierarchical re-endothelialization within whole larynxes. This is to our knowledge the first report of a re-endothelialized laryngeal scaffold, a crucial step in achieving vital, transplantable laryngeal grafts.(20) The introduction of MCGM allowed for endothelial maturation, as suggested by strengthened endothelial barrier and reduction in mitosis.(21) We next explored development of mucosal epithelial lining from hBCs and achieved a continuous epithelial monolayer on full thickness laryngeal mucosa formed by intercellular adherence junction. Due to the anatomical location of the larynx, orthotopic implants are prone to infection. The epithelium's primary role is a first line defense by providing a barrier. (22) Thus ex vivo development of a continuous cell layer may minimize risk of infection.(23) Presence of basal cell markers suggests the newly formed tissue could be differentiated into pseudostratified ciliated epithelium in vivo, however, a large animal transplantation model would be needed to assess in vivo epithelial differentiation of laryngeal mucosa. We further showed that intrinsic laryngeal muscle ECM could be repopulated with primary human myoblasts ex vivo. A crucial question in the field of organ engineering is the optimal maturation stage at which to transplant grafts regenerated ex vivo.(24) - We did not see myofiber formation after ex vivo culture, which was to be expected due to short duration of culture and absence of biochemical or biomechanical stimuli. By providing these stimuli upon heterotopic implantation, we achieved early differentiation to musclelike tissue. Extended in vivo maturation, would likely be needed to develop functional, more mature laryngeal muscle. The heterotopic implantation site proofed the concept of in vivo differentiation of bioengineered muscle constructs. One limitation of this model is 
that the implanted construct is in close proximity to native muscle. Noteworthy, we did not observe recipient myoblast migration into the implant.(25) An orthotropic transplantation of the bioengineered graft is preferred. However, an animal model matching the size of the donor animal would be required. The complexity of tissue architecture and cellular composition makes functional replacement of the larynx challenging. Ideally, engineered laryngeal tissue grafts could be constructed ex vivo and further matured orthotopically to replace missing laryngeal function. We achieved early tissue formation of three laryngeal compartments by introducing one uniform culture condition, a key factor in developing multicellular, ready-to-transplant tissue grafts.

\section{ACKNOWLEDGEMENT:}

The authors thank MGH Center for Skeletal Research Core for histological processing and MGH Department of Radiology for CTA image acquisition. The study was funded by the Sara and Charles Fabrikant MGH Research Scholarship, and by departmental funds

\section{DISCLOSURE STATEMENT:}

Dr. Ott is founder and stockholder of IVIVA Medical Inc. This relationship did not affect the present study. The authors Moser, Gerli, Diercks, Evangelista-Leite, Charest, Ren, Gilpin, Jank, Gershlak, Gaudette, and Hartnick have no competing interest. The funding organization had no role in the design and conduct of the study; collection, management, analysis, and interpretation of the data. $(2,26)$ 


\section{REFERENCES:}

1. Hashmi NK, Ransom E, Nardone H, Redding N, Mirza N. Quality of life and selfimage in patients undergoing tracheostomy. Laryngoscope. 2010;120 Suppl 4:S196.

2. Fuoco C, Salvatori ML, Biondo A, Shapira-Schweitzer K, Santoleri S, Antonini S, et al. Injectable polyethylene glycol-fibrinogen hydrogel adjuvant improves survival and differentiation of transplanted mesoangioblasts in acute and chronic skeletal-muscle degeneration. Skelet Muscle. 2012;2(1):24.

3. Cipriano A, Mao ML, Hon HH, Vazquez D, Stawicki SP, Sharpe RP, et al. An overview of complications associated with open and percutaneous tracheostomy procedures. Int J Crit IIIn Inj Sci. 2015;5(3):179-88.

4. Strome M, Stein J, Esclamado R, Hicks D, Lorenz RR, Braun W, et al. Laryngeal transplantation and 40-month follow-up. N Engl J Med. 2001;344(22):1676-9.

5. Farwell DG, Birchall MA, Macchiarini P, Luu QC, de Mattos AM, Gallay BJ, et al. Laryngotracheal transplantation: technical modifications and functional outcomes. Laryngoscope. 2013;123(10):2502-8.

6. Gilbert TW, Sellaro TL, Badylak SF. Decellularization of tissues and organs. Biomaterials. 2006;27(19):3675-83.

7. Kelly DJ, Azeloglu EU, Kochupura PV, Sharma GS, Gaudette GR. Accuracy and reproducibility of a subpixel extended phase correlation method to determine micron level displacements in the heart. Med Eng Phys. 2007;29(1):154-62.

8. Brown BN, Londono R, Tottey S, Zhang L, Kukla KA, Wolf MT, et al. Macrophage phenotype as a predictor of constructive remodeling following the implantation of biologically derived surgical mesh materials. Acta Biomater. 2012;8(3):978-87.

9. Maffioletti SM, Gerli MF, Ragazzi M, Dastidar S, Benedetti S, Loperfido M, et al. Efficient derivation and inducible differentiation of expandable skeletal myogenic cells from human ES and patient-specific iPS cells. Nat Protoc. 2015;10(7):941-58. 
10. Gilpin SE, Charest JM, Ren X, Tapias LF, Wu T, Evangelista-Leite D, et al. Regenerative potential of human airway stem cells in lung epithelial engineering. Biomaterials. 2016;108:111-9.

11. Ren X, Tapias LF, Jank BJ, Mathisen DJ, Lanuti M, Ott HC. Ex vivo non-invasive assessment of cell viability and proliferation in bio-engineered whole organ constructs. Biomaterials. 2015;52:103-12.

12. Fuoco C, Rizzi R, Biondo A, Longa E, Mascaro A, Shapira-Schweitzer K, et al. In vivo generation of a mature and functional artificial skeletal muscle. EMBO Mol Med. $2015 ; 7(4): 411-22$.

13. Guyette JP, Charest JM, Mills RW, Jank BJ, Moser PT, Gilpin SE, et al. Bioengineering Human Myocardium on Native Extracellular Matrix. Circ Res. 2016;118(1):56-72.

14. Sophia Fox AJ, Bedi A, Rodeo SA. The basic science of articular cartilage: structure, composition, and function. Sports Health. 2009;1(6):461-8.

15. Ren X, Moser PT, Gilpin SE, Okamoto T, Wu T, Tapias LF, et al. Engineering pulmonary vasculature in decellularized rat and human lungs. Nat Biotechnol. 2015;33(10):1097-102.

16. Carter EP, Olveczky BP, Matthay MA, Verkman AS. High microvascular endothelial water permeability in mouse lung measured by a pleural surface fluorescence method. Biophys J. 1998;74(4):2121-8.

17. Baiguera S, Gonfiotti A, Jaus M, Comin CE, Paglierani M, Del Gaudio C, et al. Development of bioengineered human larynx. Biomaterials. 2011;32(19):4433-42.

18. Ansari T, Lange P, Southgate A, Greco K, Carvalho C, Partington L, et al. Stem CellBased Tissue-Engineered Laryngeal Replacement. Stem Cells Transl Med. 2017;6(2):677-87.

19. Wang EC, Damrose EJ, Mendelsohn AH, Nelson SD, Shintaku IP, Ye M, et al. Distribution of class I and II human leukocyte antigens in the larynx. Otolaryngol Head Neck Surg. 2006;134(2):280-7. 
20. Pellegata AF, Tedeschi AM, De Coppi P. Whole Organ Tissue Vascularization: Engineering the Tree to Develop the Fruits. Front Bioeng Biotechnol. 2018;6:56.

21. Beaulieu Leclerc V, Roy O, Santerre K, Proulx S. TGF-beta1 promotes cell barrier function upon maturation of corneal endothelial cells. Sci Rep. 2018;8(1):4438.

22. Zhang $\mathrm{H}, \mathrm{Fu}$ W, Xu Z. Re-epithelialization: a key element in tracheal tissue engineering. Regen Med. 2015;10(8):1005-23.

23. Hamilton NJI, Birchall MA. Tissue-Engineered Larynx: Future Applications in Laryngeal Cancer. Curr Otorhinolaryngol Rep. 2017;5(1):42-8.

24. Ott HC, Clippinger B, Conrad C, Schuetz C, Pomerantseva I, Ikonomou L, et al. Regeneration and orthotopic transplantation of a bioartificial lung. Nat Med. 2010;16(8):927-33.

25. Sicari BM, Rubin JP, Dearth $\mathrm{CL}$, Wolf MT, Ambrosio F, Boninger M, et al. An acellular biologic scaffold promotes skeletal muscle formation in mice and humans with volumetric muscle loss. Sci Transl Med. 2014;6(234):234ra58.

26. Maffioletti SM, Sarcar S, Henderson ABH, Mannhardt I, Pinton L, Moyle LA, et al. Three-Dimensional Human iPSC-Derived Artificial Skeletal Muscles Model Muscular Dystrophies and Enable Multilineage Tissue Engineering. Cell Rep. 2018;23(3):899-908. 


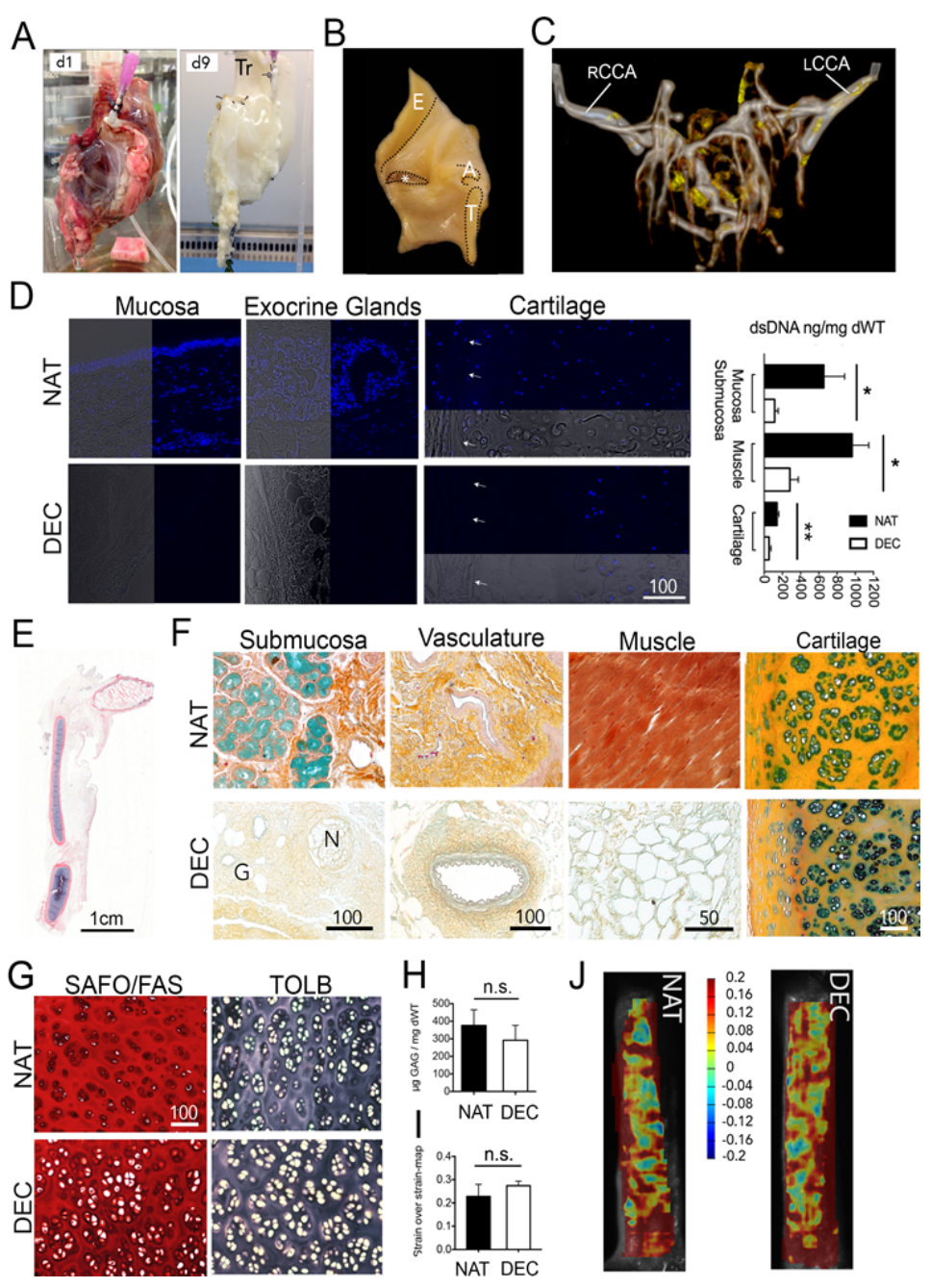

Figure 1: Creation of whole laryngeal scaffolds from native canine larynxes

(A) Photograph of an isolated whole canine larynx mounted up-side down via the trachea (Tr) and undergoing perfusion-decellularization. Day 1(d1) vs. day 9 (d9). (B) Photograph of the laryngeal luminal surface after completion of decellularization. E: Epiglottis; A: Arytenoid cartilage; T: Thyroid cartilage; *True vocal folds. (C) CT Angiogram of a decellularized larynx. Contrast agent was perfused via right and left common carotid artery (RCCA and LCCA). (D) Left panel: DAPI stain of mucosa, exocrine glands and cartilage from NAT and DEC laryngeal tissue. Overlay: Phase contrast image of the stained tissue area. Arrows: Indicate transition from perichondrium to cartilage. Right panel: dsDNA Quantification in mucosa/submucosa, muscle and cartilage from NAT and DEC laryngeal tissue. Presented as ng/mg dry weight (dWT). (E) H\&E stain of an axial section of a DEC 
28 larynx. (F) Russel-Movat pentachrome stain of various laryngeal tissue compartments. Left to right: Microarchitecture of submucosa, vasculature, muscle and cartilage of NAT and DEC laryngeal tissue. G: Exocrine glands; N: nerve. (G) Qualitative assessment of GAG content and distribution. NAT and DEC cartilage stained with Safranin-O/ Fas green (left) and Toluidin blue (right). (H) Quantitative assessment of of GAG content in NAT and DEC cartilage. Presented as $\mu \mathrm{g} / \mathrm{mg}$ dWT. (I) Strain-over-strain map of NAT and DEC cartilage under unidirectional strain. (J) Color map of representative NAT and DEC cartilage samples under strain. $* p<0.05, * * p<0.01$. Scale bars in $\mu \mathrm{m}$ except $\mathbf{E}$. 

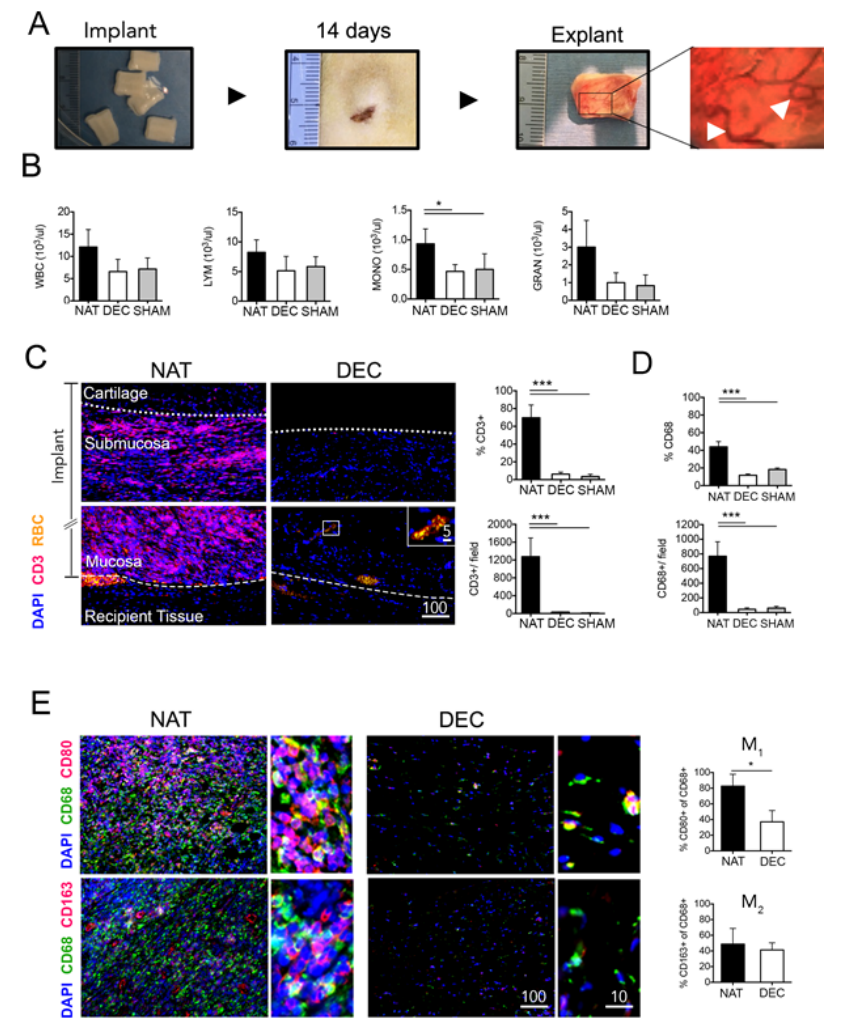

Figure 2: Immunogenic response to laryngeal scaffolds.

(A) NAT and DEC tracheal xenografts subcutaneously implanted in SD rats for 14 days. Arrowhead: Neovascularization of a DEC implant on d14. (B) Blood count $\left(10^{3}\right.$ cells/ $\left.\mu \mathrm{l}\right)$ on d5 of implantation. Left to right: Total white blood count (WBC), lymphocyte count (LYM), monocyte count (MONO) and granulocyte count (GRAN). SHAM served as controls. (C) Left panel: Immunofluorescence stain of T-lymphocyte infiltration (CD3) and vascularization (Red blood cells, RBCs) of NAT and DEC implants on d14. Insert: Proof of neovascularization. Right panel: Proportion (\%) of CD3 positive cells within mucosa / submucosa of implants (top) and absolute number of CD3 positive cells per 20x field (bottom). (D) Proportion (\%) of macrophages (CD68) within mucosa / submucosa of implants (top) and absolute number of CD68 positive cells per 20x field (bottom). (E) Left panel: Immunofluorescence stain of macrophage subpopulations (M1: $\mathrm{CD}^{+} 8^{+} / \mathrm{CD}^{\circ} 0^{+}$and M2: $\mathrm{CD}^{+} 8^{+} / \mathrm{CD} 163^{+}$) in submucosa of NAT and DEC implants on d14. Right panel: Proportion (\%) of M1 (top) and M2 (bottom) subpopulation of all CD68+ cells detected within mucosa / submucosa of implants on day $14 .{ }^{*} p<0.05,{ }^{* *} p<0.005$. Scale bars in $\mu \mathrm{m}$. 

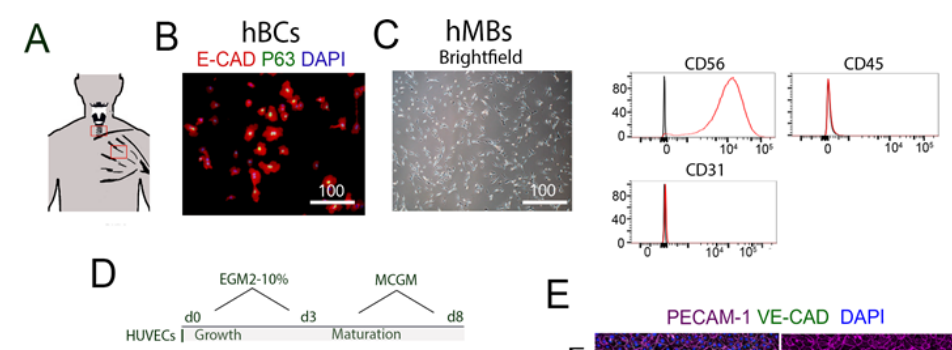

$\mathrm{E}$
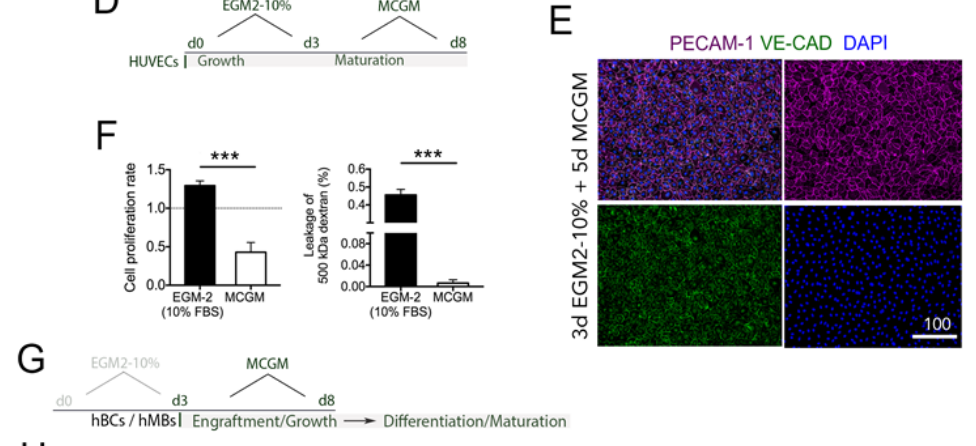

$\mathrm{F}$
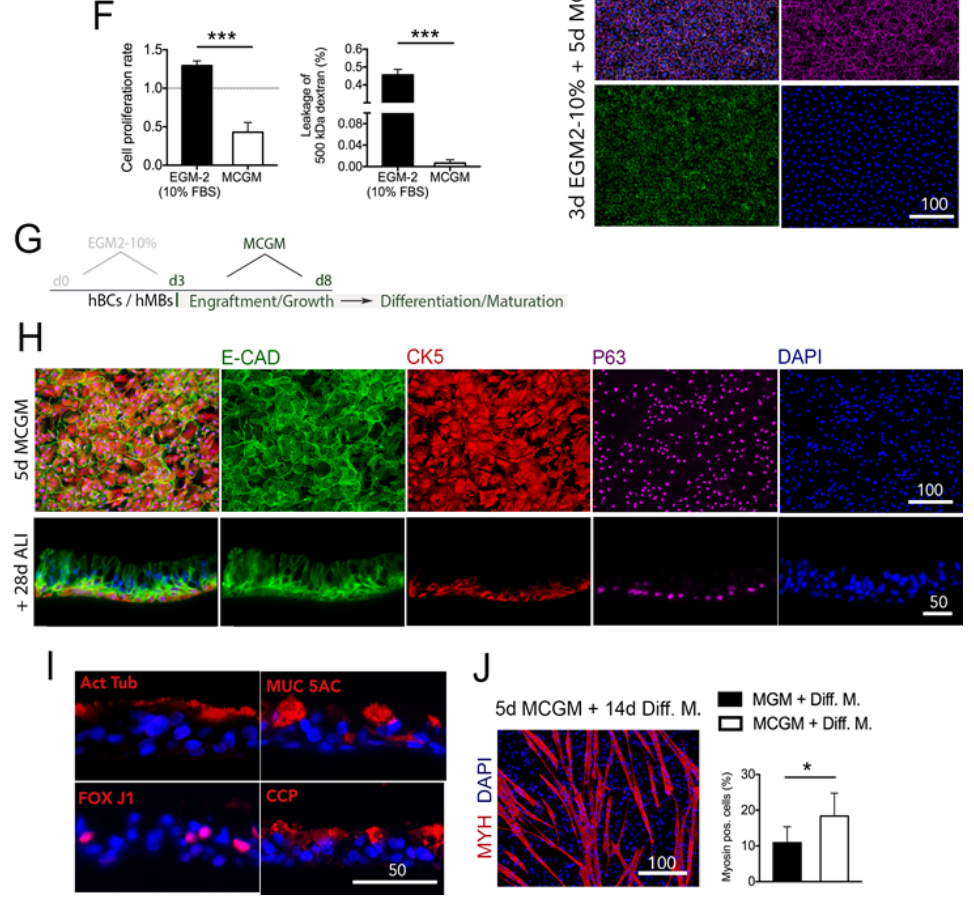

Figure 3: Development of a uniform culture condition for bioengineering of laryngeal grafts.

(A) Diagram of human primary cell isolation sites. hBCs were isolated from fresh cadaveric tracheal segments and hMBs were isolated from skeletal muscle biopsies.

(B) Epithelial-oriented culture of a pure hBC population (E-Cadherin and P63) at passage 2 (P2). (C) Left panel: Bright field image of hMBs cultured in myoblast-oriented growth medium (P2). Right panel: Flow cytometry panel of isolated hMBs positive for CD56 and negative for CD45 and CD31. (D) Schematic overview of two-step endothelialization protocol. HUVECs are seeded and cultured for 3d in EGM-2 supplemented with 8\% FBS (EGM2-10\%) followed by $5 \mathrm{~d}$ in custom formulated Multi-Cellular Growth Medium (MCGM). (E) Characterization of HUVECs cultured in EGM2-10\% until confluent on day 3, 
followed by MCGM for 5 days. HUVECs are positive for PECAM-1, VE-Cadherin and DAPI. (F) Left panel: Proliferation rate of HUVECs cultured in EGM2-10\% or MCGM and normalized to conventional EGM-2. Right panel: Percentage of 500 kDa Dextran leakage of HUVEC monolayers cultured in EGM2-10\% or MCGM and normalized to unseeded controls. (G) Schematic overview of hBC and hMBs cultured in MCGM over 5 days. (H) Characterization of hBCs cultured in MCGM. Immunofluorescence stain for E-Cadherin, CK5, P63 and DAPI of hBCs cultured for 5d in MCGM. Presence of a basal cell layer in pseudo-stratified epithelium following differentiation in air-liquid interface (ALI) for 28d (lower panel). (I) Proof of presence of mature cell types within the pseudostratified epithelium: Ciliated cells (Act Tub $\left.{ }^{+}, \mathrm{FOX} \mathrm{J1} 1^{+}\right)$, goblet cells $\left(\mathrm{MUC} 5 \mathrm{AC}^{+}\right)$and club cells $\left(\mathrm{CCP}^{+}\right)$. (J) Characterization of hMBs cultured in MCGM: Left panel: Immunofluorescence stain for Myosin heavy chain $\left(\mathrm{MYH}^{+}\right.$) positive fibers cultured for $5 \mathrm{~d}$ in MCGM, followed by $14 \mathrm{~d}$ differentiation in conventional differentiation medium (Diff. M.). Right panel: Percentage (\%) of MYH positive cells after differentiation. ${ }^{* *} \mathrm{p}<0.005$. Scale bars in $\mu \mathrm{m}$. 

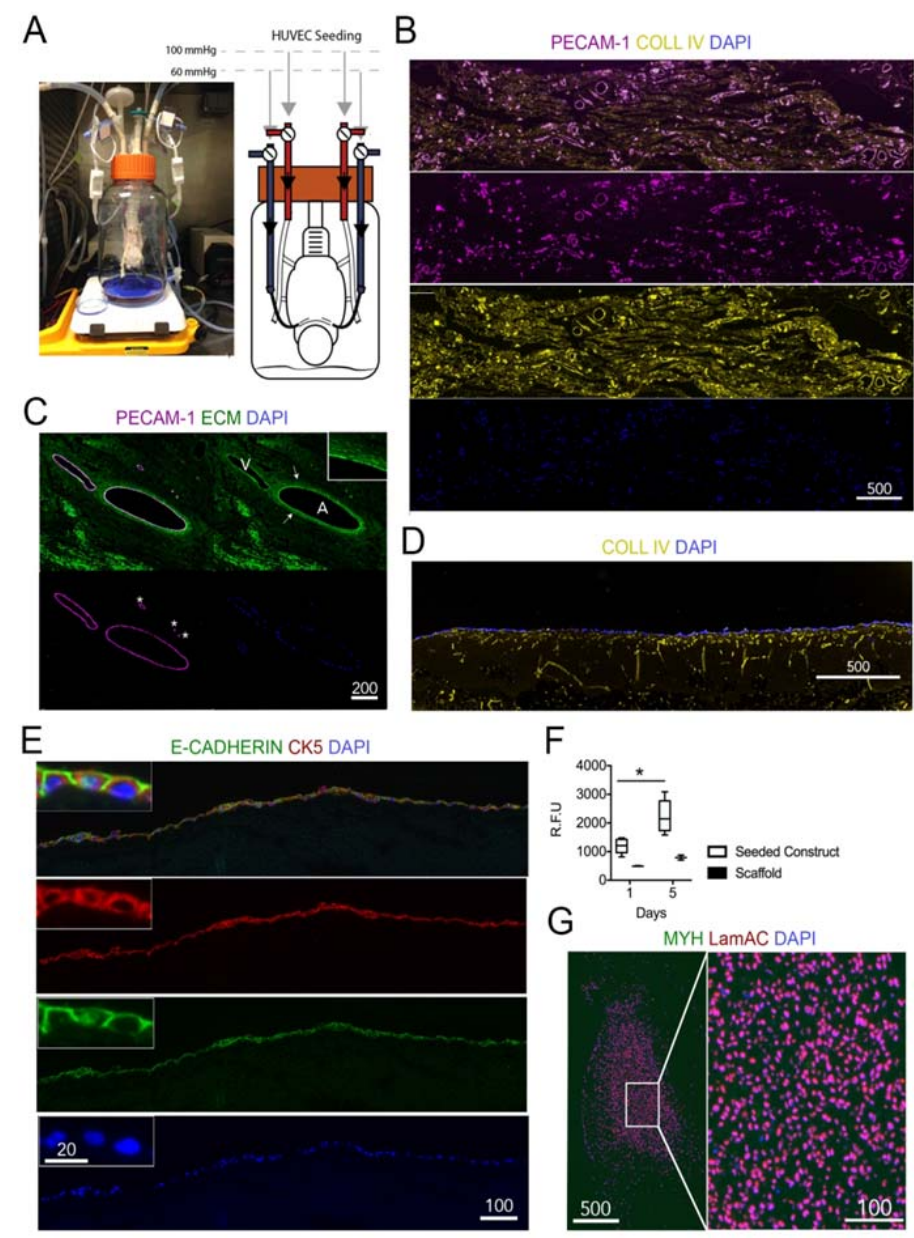

\section{Figure 4: Bioengineering of laryngeal grafts}

(A) Schematic drawing of simultaneous HUVEC seeding from two arterial (red) and two venous (blue) vascular accesses at 100 and $60 \mathrm{mmHg}$, respectively. (B) Large scale immunofluorescence image of endothelial (PECAM-1 ${ }^{+}, \mathrm{DAPI}^{+}$) coverage in a whole laryngeal scaffold (Coll IV ${ }^{+}$) after culture in EGM2-10\% for 3d followed by MCGM for $5 d$. (C) Immunofluorescence image of endothelial (PECAM- $1^{+}, \mathrm{DAPI}^{+}$) lining of an artery $(\mathrm{A})$, vein (V) and small diameter vessels $\left({ }^{*}\right)$ on day 8 of perfusion-culture. Arrows and insert show elastic fibers within the tunica media, indicating presence of an decellularized artery. (D) Large scale immunofluorescence image of $\mathrm{hBC}\left(\mathrm{DAPI}^{+}\right)$coverage of laryngeal full-thickness mucosa (Coll $\mathrm{IV}^{+}$) after $5 \mathrm{~d}$ culture in MCGM. (E) Immunofluorescence image of a continuous basal cell monolayer positive for E-Cadherin, CK5 and DAPI. (F) Change in absolute fluorescence of metabolized resazurin in muscle constructs over $5 \mathrm{~d}$ of culture. 
White box plots: hMB seeded scaffolds. Black box plots: unseeded controls. (G) Immunofluorescence image of human lamin AC (LamAC) positive cells within laryngeal muscle constructs cultured in MCGM for $5 \mathrm{~d}$. Image shows absence of MYH on day 5 . $\underset{E}{\stackrel{\mathscr{E}}{t}} \quad * p<0.05$. Scale bars in $\mu \mathrm{m}$ G) 


\section{A}

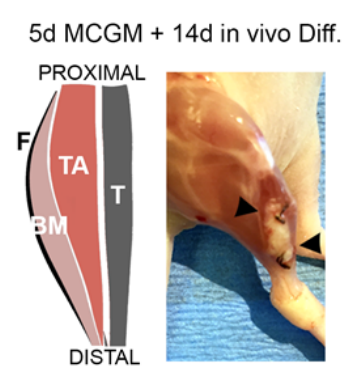

B

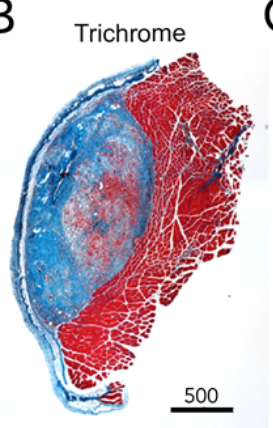

C
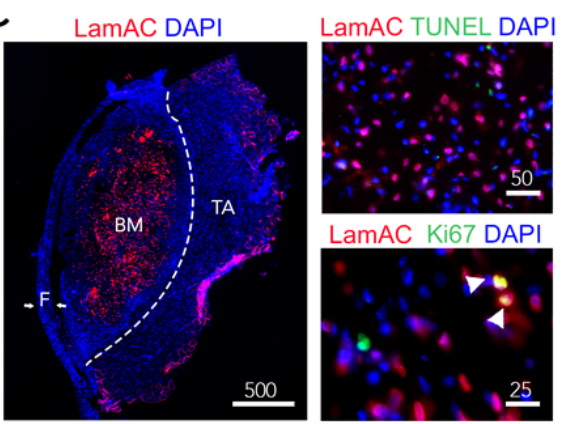

LamAC Ki67 DAPI
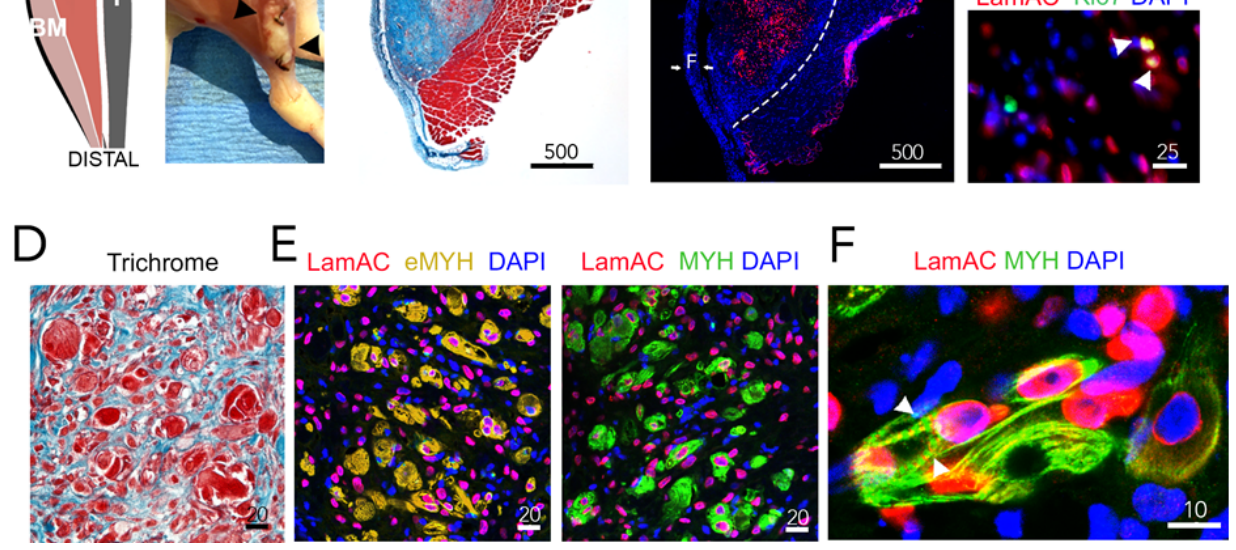

Figure 5: In vivo maturation of bioengineered laryngeal grafts

(A) Left panel: Diagram of the implantation site of bioengineered muscle (BM): The graft was placed between fascia (F) and anterior tibial muscle (TA); T: tibia. Right panel: Macroscopic appearance of the bioengineered muscle after $14 \mathrm{~d}$ of in vivo differentiation. (B) Trichrome stain of the whole implant at time of explantation. (C) Left panel: Immunofluorescence stain of seeded myoblasts $\left(\operatorname{LamAC}^{+}\right)$within the border of the BM. Right panel: Absence of apoptosis (TUNEL ${ }^{+} / \operatorname{LamAC}^{+}$) and presence of cell proliferation $\left(\mathrm{KI} 67^{+} / \mathrm{LamAC}^{+}\right)$within the BM. (D) Trichrome stain of newly formed muscular constructs with appearance of early developmental multinucleated fibers. (E) Left panel: BM positive for embryonic MYH (eMYH). Right panel: BM positive for MYH. (F) Immunofluorescence stain of a muscle fiber with early myosin striation. Scale bars in $\mu \mathrm{m}$ 


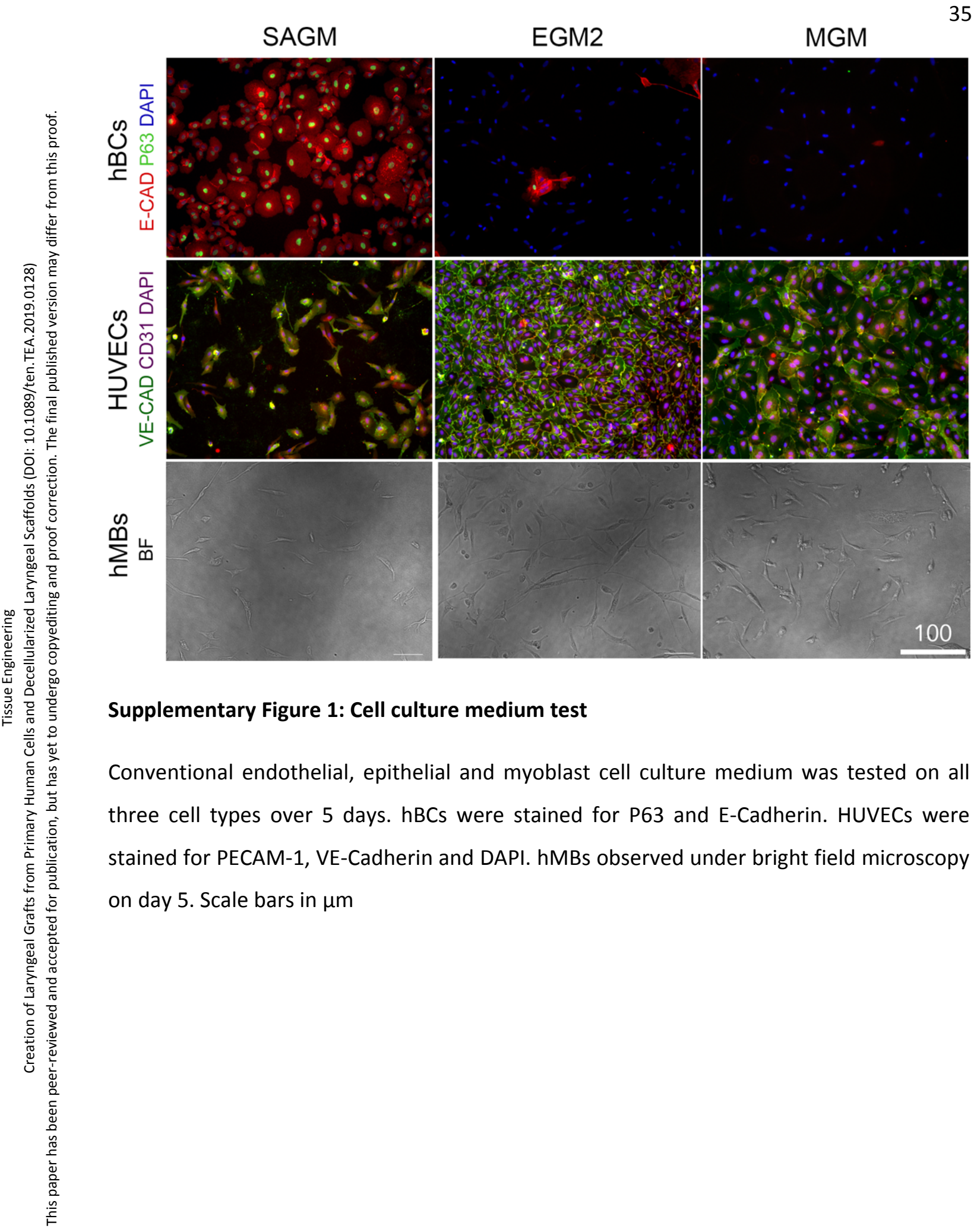




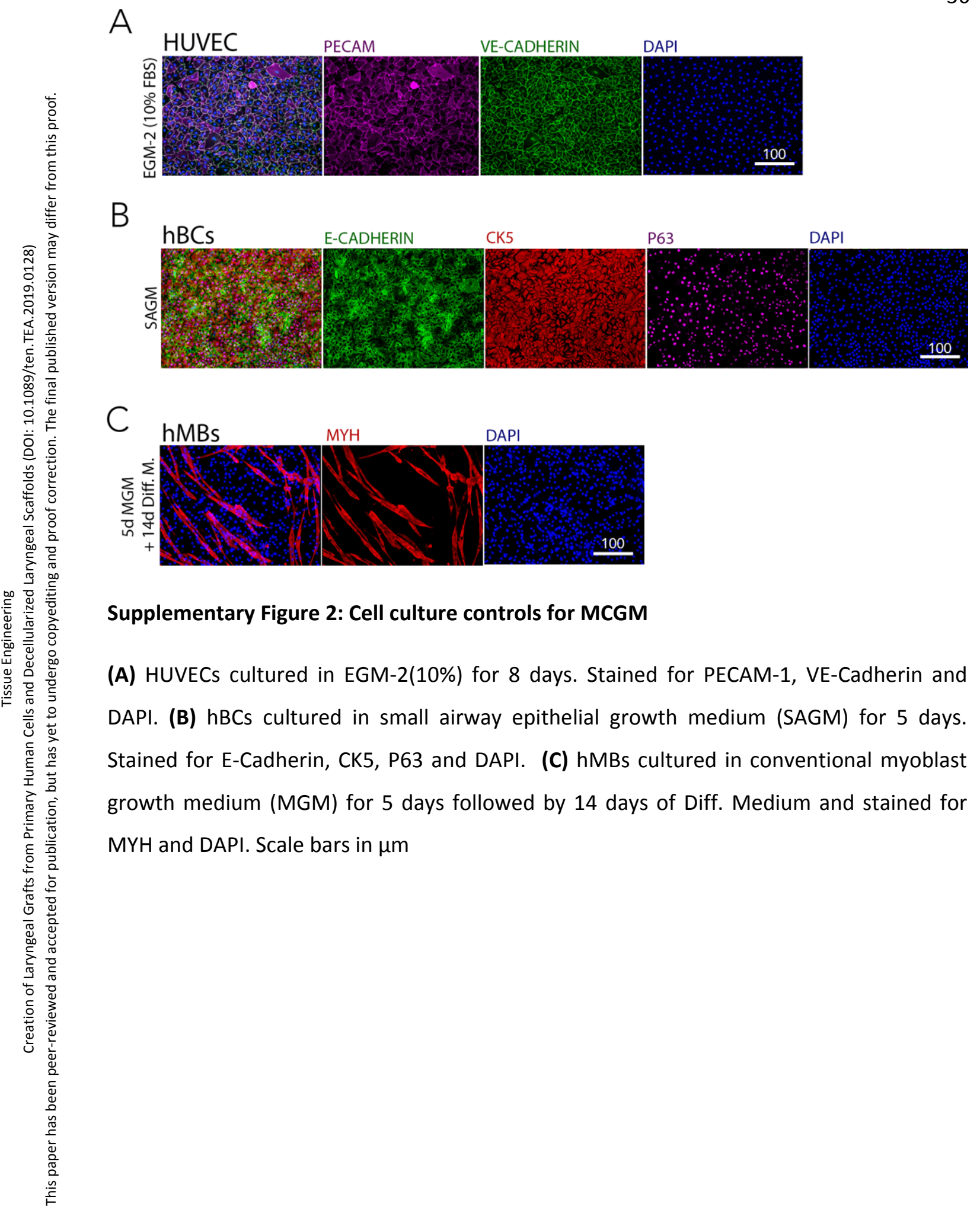


MYH LAMAC DAPI

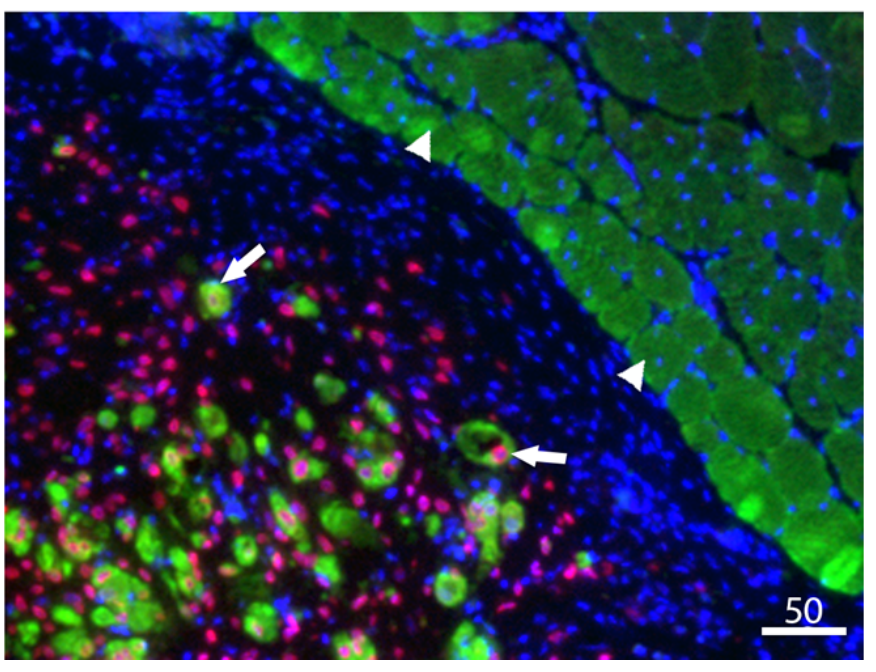

\section{Supplementary Figure 3: Laryngeal muscle implant}

Border-zone of bioengineered muscle (BM) and native tibialis anterior muscle. Small native regenerative fibers can be observed at border to implant (arrow head). Human origin identifying LamAC ${ }^{+}$were not found beyond the limits of the implant. Vice versa $\mathrm{MYH}^{+}$cells within the implant are of human origin. Scale bars in $\mu \mathrm{m}$ 